\title{
INSTRUMENTATION \& TECHNIQUES Evaluation of a sound-generator chip: The AY 3-8910
}

\author{
JAMES A. BALLAS and JOHN HOUSTON \\ Georgetown University, Washington, $D C$
}

\begin{abstract}
Despite the continuing decrease in computer prices, psychoacoustic research is still expensive because of the high cost of sound-generation equipment and software. A single-chip sound generator was evaluated as an inexpensive tone/noise generator, amplitude attenuator, envelope modulator, and sound mixer. Although the chip has some limitations, it offers important advantages for pilot research and laboratory demonstrations.
\end{abstract}

The dramatic drop in computer prices has made computerized laboratories commonplace. In a psychoacoustic laboratory, the computer is used to generate various acoustic signals, including tones and noise. Unfortunately, the hardware and software costs for these functions are still not commensurate with the cost of small computer systems. The generation of signals using digital-to-analog conversion is relatively expensive and technically difficult, often requiring a digital-toanalog (D/A) converter, a programmable clock, an analog-to-digital converter, and either a fast disk or a large memory. As an alternative, several single-chip devices that are designed as general-purpose signal generators are available. One of these is the AY 3-8910, recently described by Polich, Fischer, and Starr (1983). This chip is programmable and, as illustrated in Figure 1, offers most of the functions needed for psychoacoustic research, including tone and noise generation, amplitude attenuation, envelope modulation, and sound mixing. The advantages and limitations of this device are reported here. General information on programming and interfacing is reported by Polich et al. (1983).

\section{TONE GENERATION}

Three channels, used separately or in combination, mediate the generation of tones. Each channel is capable of producing square waves (out of phase with the other channels) at frequencies ranging from $122 \mathrm{~Hz}$ to $500 \mathrm{kHz}$ if an $8-\mathrm{MHz}$ timing clock is used. The specifications call for a maximum clock rate of $2 \mathrm{MHz}$, but the frequency resolution at this rate was unacceptable, and the clock was increased to $8 \mathrm{MHz}$ with no noticeable malfunctioning. The manufacturing practice of "margining" entails specification of the lower bounds of performance, and a chip therefore may work at speeds beyond

The authors wish to acknowledge the assistance of Ray Shaw in the evaluation of this chip. Requests for reprints should be sent to James A. Ballas, Department of Psychology, Georgetown University, Washington, DC 20057.

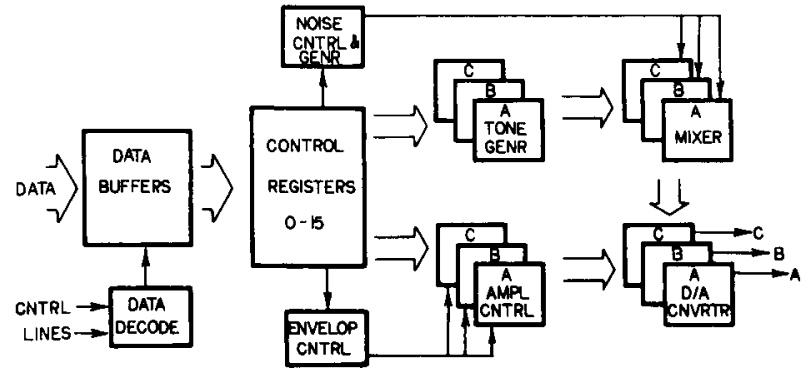

Figure 1. Functional block diagram of the AY 3-8910.

the specifications. However, the better performance is not guaranteed. The faster rate was explored because the logic used in generating tones does not allow every frequency within the range. For example, at $1000 \mathrm{~Hz}$ with an $8-\mathrm{MHz}$ clock, the frequency resolution is $2 \mathrm{~Hz}$. With a $2-\mathrm{MHz}$ clock, the resolution is only $8 \mathrm{~Hz}$. Lower frequencies yield finer frequency resolution. The frequency resolution of this device with an $8-\mathrm{MHz}$ clock is compared with typical just-noticeable-difference (jnd) data in Figure 2. It is clear that this device is appropriate

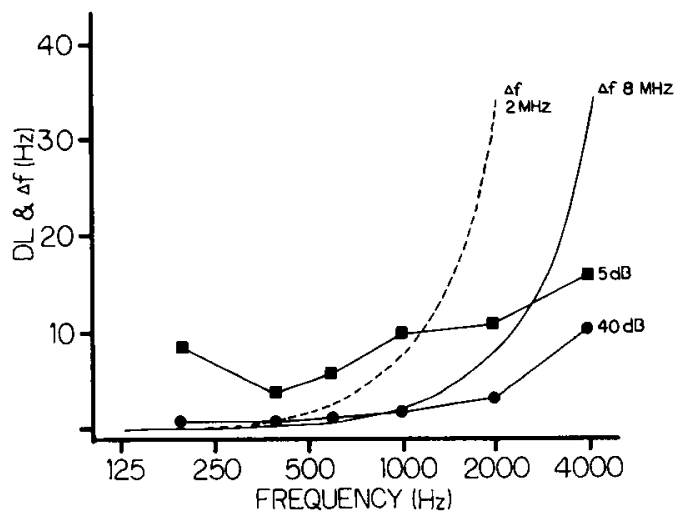

Figure 2. Frequency resolution $(\Delta f)$ of the $A Y$ 3-8910 at 2 - and $8-\mathrm{MHz}$ clock rates and frequency difference limens (Gelfand, 1981) at sensation levels of 5 and $40 \mathrm{dBs}$. 
for frequency-discrimination research only for tones under $1000 \mathrm{~Hz}$ and under $40 \mathrm{~dB}$. If tones are reduced to $5 \mathrm{~dB}$, then one may go up to $2000 \mathrm{~Hz}$.

\section{NOISE GENERATION}

One noise generator that can be mixed into any of the three channels is provided. This generator produces a square wave that varies in periodicity according to microprogrammed logic internal to the chip. Some control over the frequency composition of the noise is provided through a noise-period register. It appears that this register defines the low-pass cutoff of the noise, but not as the cutoff is traditionally defined, that is, as the one-half power or $-3 \mathrm{~dB}$ level. Rather, spectrum analyses indicate that the cutoff is approximately $-25 \mathrm{~dB}$, as depicted in Figure 3.

Use of the noise generator requires caution. The digital source of the noise is apparently a repetitive algorithm that introduces noticeable, repetitive "glitches" into the noise. These vary according to the value of the noise period and are sometimes reduced to a level that would not interfere with demonstrations. These transients are not eliminated by filtering and are not reduced by using a $2-\mathrm{MHz}$ clock. Thus, this noise generator appears to be unacceptable for serious acoustic research. One advantage of the generator is that the upper cutoff of the noise can be modified quickly and easily, thereby making it possible to do research on frequency glides using noise rather than tones.

\section{AMPLITUDE ATTENUATION}

The output of each of the three channels is through $\mathrm{D} / \mathrm{A}$ converters. The square-wave amplitude is controlled by a 4-bit register, thus providing 16 levels of amplitude. When the register is cleared, the channel is effectively disabled, and therefore the remaining 15 levels denote the degree of amplitude attentuation. The D/A converters are designed to respond on an approximately logarithmic scale to the amplitude control values, as shown in Figure 4. Notice that the middle values are

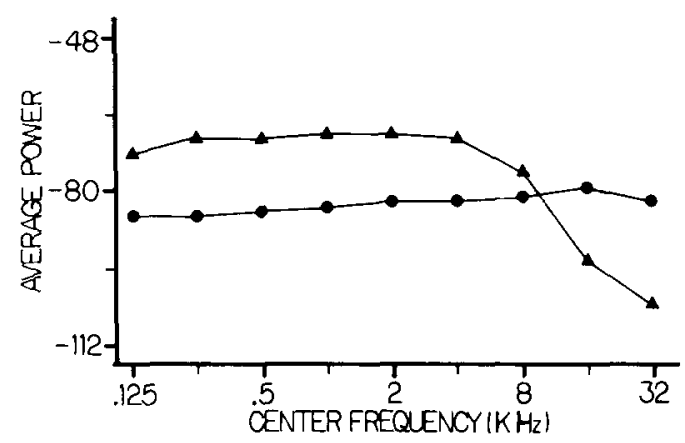

Figure 3. Average power per cycle of the noise generator for programmed noise frequencies of $80 \mathrm{kHz}$ (circles) and $16 \mathrm{kHz}$ (triangles).

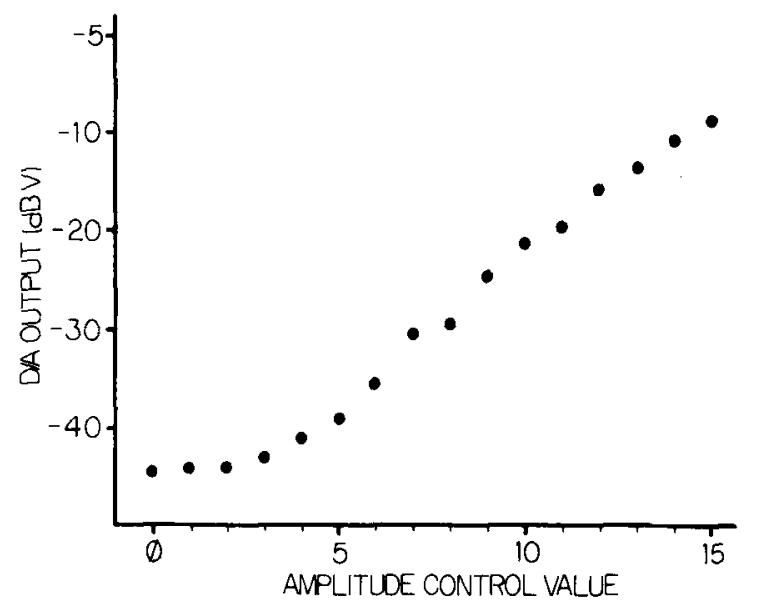

Figure 4. D/A output level as a function of the amplitude control value.

not precisely consistent with the $\log$ function. Except for these deviations, the logarithmic response is nicely suited for psychophysical scaling research. Fifteen levels is adequate for many purposes, including category and magnitude scaling of loudness.

The amplitude control is independent for each of the three channels. An increased range is possible by combining the outputs of the channels and setting each channel for maximum amplitude. Levels below this maximum can be obtained by modifying the levels within each channel. However, since the three channels are not in phase, the result depends upon the frequency that is being generated.

\section{ENVELOPE MODULATION}

The sound source, tone and/or noise, can be modulated using the envelope function. Exponential rise and decay shaping is possible, with the period controllable over a range from $256 /$ clock to $16776 /$ clock. With an $8-\mathrm{MHz}$ clock, this range is from 32 microsec to $2.09 \mathrm{sec}$. Exponential rise and decay enveloping can be used to reduce onset and offset transients and offers some advantages over the linear rise/decay modulation that is typically used (Victoreen, 1974).

The envelope function provides several types of modulation. These include a single decay or attack period, followed by either full amplitude or full attenuation. Two other alternatives include cyclic attack or decay and alternation between attack and decay. Each of these options produces a periodically modulated sound. If the modulated source is noise, then the sound will be heard as "zipping," "hammering," or "sandpapering," depending on the duration of attack and decay. Sounds such as these have been used to simulate underwater propeller cavitation in research on environmental sound perception (Howard \& Ballas, 1983).

This function has two limitations: (1) Envelope modulation precludes amplitude control. The envelope 
function is actually a preprogrammed form of amplitude control, and there is no option to specify that the amplitude should rise to less than the full value. (2) The function does not permit holding the amplitude at a constant level for some time period between the rise and decay cycles. This feature, were it included, would enable one to easily "window" pure tones. In a research setting, this function is of little use unless impact or explosive sounds are of interest. The rise and decay modulation can be used to eliminate onset and offset transients, but control over the amplitude level is sacrificed.

\section{SOUND MIXTURE}

Tones and noise can be produced and mixed on each channel. Three separate mixers enable or disable four sound sources independently (see Figure 1). These are the tone generators for each channel and a noise generator common to all channels. The tone frequencies of the three channels are independent and are specified by the respective frequency-control registers. However, there is only one noise source, and this is simply duplicated if it is selected for more than one channel.

Mixture within a channel of tone and noise occurs within the chip, whereas mixture of the separate channels depends on whether they are wired together using standard passive-mixing circuits. External mixture of the three channels provides the capability of producing complex signals with up to three programmable tonal components. Keeping the channels separate provides the capability of producing binaural stimuli. Therefore, a wide variety of psychoacoustic phenomena that require multiple sound sources can be produced. If the channels are combined externally, then monaural phenomena such as beats, tonal masking, periodicity pitch, and loudness summation can be demonstrated. If two channels are delivered separately to each ear, then binaural phenomena such as loudness summation, masking-level difference, binaural beats, and localization can be produced. We have verified each of these examples.

External mixture of the separate channels offers an important advantage due to the nature of the enable function. Let us assume that the desired stimuli are to be delivered successively and that the time intervals are too short to program all of the changes. In this case, each channel can be programmed to produce a different stimulus. The successive stimuli are generated by enabling and disabling each channel in turn. Since channels and sound sources within channels are all selected through a single control register, changes require only a single register-load operation. This feature makes it possible to produce within milliseconds successive stimuli that vary on several parameters. Furthermore, the programming of a disabled channel does not interfere with the ongoing output of an enabled channel. Thus, while one channel is playing, the parameters for the other channels can be programmed.

In conclusion, there are some advantages and disadvantages in using this device. The flexibility and versatility inherent in the five available functions, all under some form of programmable control, can be of substantial importance for both research and teaching. For pilot testing, the convenience of the system means that a signal or pattern of signals can be generated quickly and easily. (For example, auditory streaming, which can require complex tonal sequences, can be demonstrated with a few commands.) However, the limits on the frequency resolution and the periodic glitches in the noise are characteristics that may preclude the use of this device for some research.

\section{REFERENCES}

Gelfand, S. A. (1981). Hearing: An introduction to psychological and physiological acoustics. New York: Dekker.

Howard, J. H., Jr., \& Ballas, J. A. (1983). Perception of simulated propeller cavitation. Human Factors, 25, 643-655.

Polich, J., Fischer, A., \& Stark, A. (1983). A programmable multiple-tone generator. Behavior Research Methods \& Instrumentation, 15, 39-41.

Victoreen, J. A. (1974). Equal loudness pressures determined with a decaying oscillatory waveform. Journal of the Acoustical Society of America, 55, 309-312.

(Manuscript received August 4, 1983; revision accepted for publication April 10,1984.) 